Forum 2018 · 33:269

https://doi.org/10.1007/s12312-018-0461-9

Online publiziert: 14. August 2018

(c) Springer Medizin Verlag GmbH, ein Teil von

Springer Nature 2018

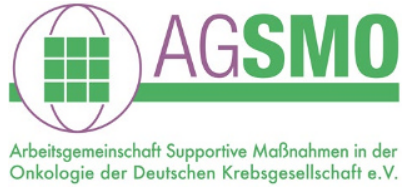

Supportive Maßnahmen sind essentielle Bausteine einer modernen Tumortherapie, denn erst sie ermöglichen die Durchführung der nebenwirkungsreichen onkologischen Therapiekonzepte ohne Dosiskompromisse oder Therapieunterbrechungen bei Erhalt der Lebensqualität der Patientinnen und Patienten. Die kontinuierliche Fort- und Weiterbildung ist dabei essenziell, denn die Onkologie unterliegt einem ständigen Wandel mit neuen Therapiekonzepten, die auch neue supportive Konzepte erfordern.

Bereits vor fast 20 Jahren hat der Verein AK Suppo, später ASO (Arbeitskreis Supportive Maßnahmen in der Onkologie) diese Notwendigkeit erkannt und mit dem Springer Verlag und seiner Zeitschrift „Im Focus Onkologie“ eine Plattform gefunden, die uns die kontinuierliche Weiterbildung in schriftlicher Form $10 \mathrm{Mal}$ im Jahr ermöglicht. Der erste Beitrag des AK Suppo erschien schon im Jahr 2000 zum Thema Antiemese.

Im Jahr 2008 haben sich der Arbeitskreis Supportive Maßnahmen in der Onkologie (ASO) und die damalige Arbeitsgemeinschaft Rehabilitation, Nachsorge und Sozialmedizin (ARNS) zur Arbeitsgemeinschaft „Supportive Maßnahmen in der Onkologie, Rehabilitation und Sozial-

P. Ortner

Pomme-med GmbH, München, Deutschland

\title{
Arbeitsgemeinschaft Supportive Maßnahmen in der Onkologie (AGSMO) formiert sich neu
}

medizin“ (ASORS) der Deutschen Krebsgesellschaft zusammengeschlossen. Dieser Zusammenschluss hatte zum Ziel, die Bereiche Supportivtherapie und Rehabilitation als unterstützende und begleitende Maßnahmen der aktiven Tumortherapie zu stärken und in der onkologischen Fachgesellschaft besser publik zu machen und zu etablieren. In gemeinsamen 2-jährlich stattfindenden Kongressen, Fortbildungsveranstaltungen, Symposien beim Deutschen Krebskongress sowie auch mit den kontinuierlichen Beiträgen in der als gemeinsames Standesorgan ausgewiesenen Fachzeitschrift „Im Focus Onkologie“ wurde dies in 10 Jahren gemeinsamer engagierter und partnerschaftlicher Arbeit hervorragend umgesetzt.

Nun schien es an der Zeit, die beiden Bereiche wieder in zwei eigenständige Arbeitsgruppen der Deutschen Krebsgesellschaft - Arbeitsgemeinschaft Supportive Maßnahmen in der Onkologie (AGSMO) und Arbeitsgemeinschaft für Onkologische Rehabilitation und Sozialmedizin (AGORS) - zu überführen. Diese Trennung erfolgte im Konsens mit den Vorstandsmitgliedern und den Mitgliedern bei der Mitgliederversammlung der ASORS während des Deutschen Krebskongresses 2018 in Berlin.

\section{Der neue Vorstand}

Bei der Mitgliederversammlung auf dem Deutsche Krebskongress 2018 wurde Frau Prof. Petra Feyer, Berlin zur Vorsitzenden der AGSMO gewählt. Stellvertreterin ist Frau Prof. Karin Jordan, Heidelberg. Mitglieder des Leitungsgremiums sind PD Dr. Sportwiss. F. Baumann, Dr. med. T. Behlendorf, Prof. Dr. med. I. J. Diel, Dr. med. M. Horneber, Prof. Dr. med H. Link, Dr. rer. nat. P. Ortner, Dr. med. F. Overkamp und Prof. Dr. med. H. Strik.

\) Am 15. und 16. März 2019 findet der Jahreskongress der AGSMO in Berlin statt

Bitte merken Sie den Termin vor. Weitere Informationen zur AGSMO finden Sie unter www.onkosupport.de.

\section{Korrespondenzadresse}

Dr. rer. nat. Petra Ortner

AGSMO - Öffentlichkeitsarbeit und Kommunikation c/o: POMME-med GmbH

Von-Erckert-Str. 48, 81827 München,

Deutschland

ortner@pomme-med.de 